\title{
Del trigo y la cizaña
}

\section{From wheat and tares}

Diariamente en su quehacer, el clínico se enfrenta a situaciones e interrogantes que debe resolver como parte de la atención a sus pacientes: ¿cómo deberá abordar el estudio y el proceso diagnóstico de su enfermo, ¿cuál prueba solicitar?, ¿cuál será su rendimiento?, ¿qué tan sensible y específica será?, ¿cuál debe ser la mejor estrategia de tratamiento?, ¿cuál es la eficacia y la efectividad de los posibles tratamientos y cuál o cuáles serán más útiles para la condición de su paciente?, ¿cuál será el pronóstico?. Hace unos 30 años Covell y colaboradores (1) informaron que en promedio, los médicos internistas se plantean dos preguntas por cada tres pacientes que ven en su consulta, y una revisión sistemática de la literatura reciente encontró que los clínicos intentan responder 51\% (36-66\%) de estas preguntas, logrando aclarar $78 \%$ (67-88\%) (2).

Desde sus orígenes, la medicina basada en evidencia ha propendido por "el uso consciente, explícito y juicioso de la mejor evidencia actual en la toma de decisiones acerca del cuidado de pacientes individuales" (3) y en este orden de ideas, ha propuesto elementos de juicio para reconocer los mejores artículos sobre diagnóstico $(4,5)$, riesgo (6), pronóstico (7), análisis de decisión $(8,9)$, tratamiento $(10)$ y muchos otros temas más. La tarea del médico al respecto consiste pues, en que una vez planteadas las preguntas o interrogantes clínicos, las traduzca en estrategias de búsqueda que le lleven hacia la información de la mayor calidad posible para responderlos. No siempre es fácil cumplir el cometido, pues existen diferentes barreras, tanto en personal experto como en entrenamiento, dentro de las cuales pueden citarse entre otras, dificultades en la formulación adecuado de la pregunta clínica, incapacidad para establecer la estrategia de búsqueda apropiada, limitaciones para establecer cuándo se ha adquirido información suficiente (cuándo detener la búsqueda), falta de entrenamiento en el uso de recursos informáticos, dificultades en el acceso a los recursos de información, falta de tiempo o aun no considerar que exista una respuesta a muchos de sus interrogantes (11-14).

Si bien hoy en día, fuentes como las revisiones sistemáticas, las publicaciones secundarias basadas en evidencia (por ejemplo ACP Journal Club o Evidence Based Medicine) (15), o los recursos de revisión exhaustiva computadorizados también fundamentados en evidencia como UpToDate tienen una gran importancia en la práctica clínica (16), la consulta de artículos primarios en fuentes como PubMed, Medline, Ovid o EMBASE sigue siendo una herramienta importante $\mathrm{y}$ de gran utilidad. Dadas las limitaciones en el proceso de búsqueda que ya se mencionaron, en particular las de habilidad y tiempo, se han hecho esfuerzos por simplificar el proceso de búsqueda en estas bases y es así como se han establecido los Clinical Queries, fundamentados en el trabajo de Haynes y colaboradores $(17,18)$, los cuales consisten en filtros que combinan elementos de búsqueda preestablecidos para recuperar artículos sobre etiología, diagnóstico, tratamiento y pronóstico entre otros. La sensibilidad de dichas ayudas de búsqueda oscila entre 93 y $98 \%$ y su especificidad entre 70 y $97 \%$ para los artículos de tratamiento; mientras que son de $64-98 \%$ y de $74-98 \%$ respectivamente para los de diagnóstico, y depende de si se escoge una estrategia más sensible o más específica. Estas ayudas han demostrado ser bastante útiles en la identificación tanto de artículos primarios como de revisiones sistemáticas (17), lo cual se traduce en rendimientos competitivos, a veces incluso con una mayor velocidad en la identificación de artículos de mejor calidad que lo que puede lograrse mediante búsquedas libres hechas por el clínico empleando palabras clave y términos que éste considere apropiados (19).

El volumen de información médica publicada periódicamente en medicina interna ha crecido en forma inusitada. En 1992 se contaba con 20 revistas que daban a luz a unos 6000 artículos anuales (y que de pretender
Ver artículo: página 194

Dr. Juan Carlos Velásquez: FACP. Médico Internista, Oncólogo Clínico. Bogotá, D.C. (Colombia). Correspondencia Dr. Juan Carlos Velásquez. Bogotá, D.C. (Colombia).

E-mail: jcvelasquez.2000@gmail. com 
leerlos todos equivaldrían a revisar unos 17 artículos diarios) (15), mientras que hoy en día se ha calculado que un profesional de cuidado primario necesitaría leer un número absolutamente inalcanzable de artículos, contenidos en más de 13 millones de referencias y más de 4800 publicaciones, considerando sólo aquellas incluidas en Medline (20). Inclusive si el material se restringe únicamente a guías de práctica clínica, un médico que tenga a su cargo 18 pacientes (con un equivalente a 44 diagnósticos diferentes) deberá leer más de 3600 páginas, equivalentes a más de 120 horas de esfuerzo (21). Es por esto que seleccionar la literatura médica de verdadera calidad constituye en el presente una prioridad, al momento de buscar las respuestas a nuestras inquietudes clínicas cotidianas.

En este número de la revista, M. Álvarez y colaboradores (22), nos muestran cuál ha sido el comportamiento de la publicación de artículos que contienen elementos de calidad identificables a través de Clinical Queries en cuatro áreas de la medicina interna a saber: cardiología, endocrinología, neumología y nefrología. Los autores han encontrado que de 498952 publicaciones sobre diagnóstico y tratamiento identificadas en PubMed, $16 \%$ tienen estos elementos de calidad, siendo más frecuentes en cardiología (23\%) y endocrinología (13\%), concretamente en temas como enfermedad coronaria o infarto agudo del miocardio (75\% de los artículos de cardiología) y diabetes mellitus (61\% de los de endocrinología). Es interesante constatar cómo ha habido un aumento sustancial de este tipo de información entre los años 90 y la primera década de los años 2000, pasando de 18059 artículos a 43808 , lo que corresponde a un aumento de 2.4 veces la cifra inicial, y verificar particularmente este incremento en el área de diabetes, donde hoy por hoy hay siete veces más publicaciones con estas características. El predominio de toda esta literatura es en el idioma inglés (98\%), y sólo $2 \%$ de los artículos de esta base son publicados en español o en francés.

El panorama presentado entonces, sugiere un incremento en la calidad de la literatura publicada y recuerda al lector la disponibilidad del recurso de los Clinical Queries como un elemento de juicio de la calidad de la información, haciendo la salvedad desde luego que este es sólo un elemento para identificar los artículos que tienen una mayor posibilidad de brindar información relevante a la hora de evaluar el rendimiento y utilidad de un tratamiento o de una prueba diagnóstica, sin desconocer el valor que otro tipo de publicaciones tengan para la práctica cotidiana. Dado el volumen aun no despreciable, sigue siendo fundamental que el clínico conserve la destreza necesaria para realizar búsquedas fructíferas que proporcionen el resultado pretendido en nuestro escaso tiempo disponible (23). Por último, es preciso recordar siempre, que la intención final del esfuerzo de búsqueda, al menos en el escenario clínico, debe estar dirigida a individualizar el análisis de la evidencia en favor de nuestro paciente concreto, sopesando la aplicabilidad del conocimiento en su situación particular, discutiendo ojalá con él la verdadera utilidad que dicho conocimiento puede proporcionar en su caso real, lo cual está mucho más allá de la simple implementación en masa de unos conceptos o unas propuestas globales (24). El pan está hecho de trigo, no de cizaña.

\section{Referencias}

1. Covell DG, Uman GC, Manning PR. Information needs in office practice: are they being met? Ann Intern Med. 1985;103(4):596-9.

2. Del Fiol G, Workman TE, Gorman PN. Clinical questions raised by clinicians at the point of care: a systematic review. JAMA Intern Med. 2014;174(5):710-8.

3. Sackett DL, Rosenberg WM, Gray JA, Haynes RB, Richardson WS. Evidence based medicine: what it is and what it isn't. BMJ. 1996;312(7023):71-2.

4. Jaeschke R, Guyatt G, Sackett DL. Users' guides to the medical literature. III. How to use an article about a diagnostic test. A. Are the results of the study valid? Evidence-Based Medicine Working Group. JAMA. 1994;271(5):389-91.

5. Jaeschke R, Guyatt GH, Sackett DL. Users' guides to the medical literature. III. How to use an article about a diagnostic test. B. What are the results and will they help me in caring for my patients? The Evidence-Based Medicine Working Group. JAMA. 1994;271(9):703-7.

6. Levine M, Walter S, Lee H, Haines T, Holbrook A, Moyer V. Users' guides to the medical literature. IV. How to use an article about harm. Evidence-Based Medicine Working Group. JAMA. 1994;271(20):1615-9.

7. Laupacis A, Wells G, Richardson WS, Tugwell P. Users' guides to the medical literature. V. How to use an article about prognosis. Evidence-Based Medicine Working Group. JAMA. 1994;272(3):234-7.

8. Richardson WS, Detsky AS. Users' guides to the medical literature. VII. How to use a clinical decision analysis. A. Are the results of the study valid? Evidence-Based Medicine Working Group. JAMA. 1995;273(16):1292-5.

9. Richardson WS, Detsky AS. Users' guides to the medical literature. VII. How to use a clinical decision analysis. B. What are the results and will they help me in caring for my patients? Evidence Based Medicine Working Group. JAMA. 1995;273(20):1610-3.

10. Guyatt GH, Sackett DL, Cook DJ. Users' guides to the medical literature. II. How to use an article about therapy or prevention. B. What were the results and will they help me in caring for my patients? Evidence-Based Medicine Working Group. JAMA. 1994;271(1):59-63. 
11. Ely JW, Osheroff JA, Ebell MH, Chambliss ML, Vinson DC, Stevermer JJ, et al. Obstacles to answering doctors' questions about patient care with evidence: qualitative study. BMJ. 2002;324(7339):710.

12. Ely JW, Osheroff JA, Chambliss ML, Ebell MH, Rosenbaum ME. Answering physicians' clinical questions: obstacles and potential solutions. J Am Med Inform Assoc. 2005;12(2):217-24.

13. Green ML, Ruff TR. Why do residents fail to answer their clinical questions? A qualitative study of barriers to practicing evidence-based medicine. Acad Med. 2005;80(2):176-82.

14. van Dijk N, Hooft L, Wieringa-de Waard M. What are the barriers to residents' practicing evidence-based medicine? A systematic review. Acad Med. 2010;85(7):1163-70.

15. Davidoff F, Haynes B, Sackett D, Smith R. Evidence based medicine. BMJ. 1995;310(6987):1085-6.

16. Isaac T, Zheng J, Jha A. Use of UpToDate and outcomes in US hospitals. J Hosp Med. 2012;7(2):85-90.

17. Wilczynski NL, McKibbon KA, Haynes RB. Sensitive Clinical Queries retrieved relevant systematic reviews as well as primary studies: an analytic survey. J Clin Epidemiol.
2011;64(12):1341-9

18. Haynes RB, Wilczynski N. Finding the gold in MEDLINE: clinical queries. ACP J Club. 2005;142(1):A8-9.

19. Lokker C, Haynes RB, Wilczynski NL, McKibbon KA, Walter SD. Retrieval of diagnostic and treatment studies for clinical use through PubMed and PubMed's Clinical Queries filters. J Am Med Inform Assoc. 2011;18(5):652-9.

20. Gopalakrishnan S, Ganeshkumar P. Systematic Reviews and Meta-analysis: Understanding the Best Evidence in Primary Healthcare. J Family Med Prim Care. 2013;2(1):9-14.

21. Allen D, Harkins KJ. Too much guidance? Lancet. 2005;365(9473):1768.

22. M.Alvarez, R. Dennis, C. Villar. Características de estudios publicados en áreas del conocimiento de Medicina Interna incluyendo calidad, idioma y década de publicación. Acta Med Colomb 2015; 40: 194-201.

23. Waltho D, Kaur MN, Haynes RB, Farrokhyar $F$, Thoma A Users' guide to the surgical literature: how to perform a highquality literature search. Can J Surg. 2015;58(5):349-58.

24. Greenhalgh T, Howick J, Maskrey N. Evidence based medicine: a movement in crisis? BMJ. 2014;348:g3725. 